\title{
Some aspects of near-surface vortices
}

\author{
M Gharib \\ California Institute of Technology, Pasadena CA 91125
}

\begin{abstract}
An experimental investigation of the dynamics of near surface vortices is presented. Using digital particle image velocimetry, the velocity and vorticity fields of a vortex ring and a pair of undulated vortices were mapped as they rise toward a free surface . Circulation measurements indicate that vortex disconnection and appearance of the surface-normal vortices are simultaneous. Vortex disconnection due to the vorticity flux through the surface is shown to be related to the free-surface deceleration.
\end{abstract}

\section{INTRODUCTION}

Our understanding of turbulence near the shear-free boundaries (free surfaces) suffers from the same level of empiricism that dominates the general field of turbulence. Roshko (1992) pointed out that one impact of statistical theory of turbulence was to tend to encourage a view of "fully turbulent" flow as too complicated and disorganized to contain structural features that could be usefully incorporated into any model. However, it takes no extraordinary effort to recognize that, in general, free surface flows are dominated by vortical turbulent structures. The free-surface deformation behind a ship (with Reynolds number of $10^{8}$ or higher) provides a natural visualization of this fact. Therefore the research scene in free-surface turbulence is dominated mainly by the efforts that are focused toward understanding the interaction of the vorticity field with shear-free boundaries.

In general, we like to believe that turbulence is an ensemble of interacting vortices. Very near the free surface the elements of this ensemble may be modeled as vortex rings and vortex pairs since these model elements are self consistent from fundamental laws of vortex flows. These elemental vortex systems, once exposed to a free surface, are obliged to follow the kinematic conditions imposed by such shear-free surfaces. For the case of noslip condition the vortex filaments can only exist parallel to the surface; usually, the existence of a boundary layer provides a natural setting for such a compliance. For shear-free surfaces, vortices can locally connect to the free surface at a normal angle. The preceding contrast between the two boundary conditions and the associated behavior of vortices require different approaches for capturing the essential mechanisms of free-surface turbulence.

All the relevant free surface flows such as ship wakes are complex, which means full simulation of them (numerical or experimental) is difficult and often not very useful until a good deal of physical understanding would be developed. Therefore, a natural approach would be to break down the problem into more elemental flows such as isotropic turbulence, jets, mixing layers and, on a more elementary level, vortex pairs and rings. In this article we will concentrate mainly on some important aspects of vortex interaction with free surfaces and try to shed some light on the nature of vorticity flux through the free surface.

\section{VORTEX INTERACTION WITH A FREE SURFACE}

The first clear demonstration of the early stages that a vortex ring undergoes as it rises toward a free surface was made by Bernal and Kwon (1989). They showed that as a vortex ring approaches the free surface at an oblique angle, the apex part of the vortex ring deforms and opens, disconnecting its ends to reconnect to the surface. While the process of normal reconnection of the ends to the free surface can be explained by the kinematic condition at the free surface, the faith of the segment of the vortex that first approaches the surface and then disappears has been the subject of many debates. Rood (1994a) has suggested that the disconnection process is due to the outward flux of surface-parallel vorticity through the viscous acceleration of the free-surface in a direction tangential to the free surface. Rood's mathematical formulation provided a physical basis for the modeling of this intriguing process. In order to understand this process, we need to examine the vorticity flux equation in the form suggested by Rood:

$$
v\left(\frac{\partial \omega_{z}}{\partial y}\right)=-\frac{\partial u_{s}}{\partial t}+\frac{\partial H}{\partial S},
$$

where $\omega_{Z}$ is the surface-parallel vorticity, $u_{s}$ the surface velocity, and $H$ the total head. In this formulation, the surface acceleration $\left(\frac{\partial u}{\partial t}\right)_{S}$ in the absence of large surface deformation would be the leading term. This leads to an approximate version of Rood's formula: 

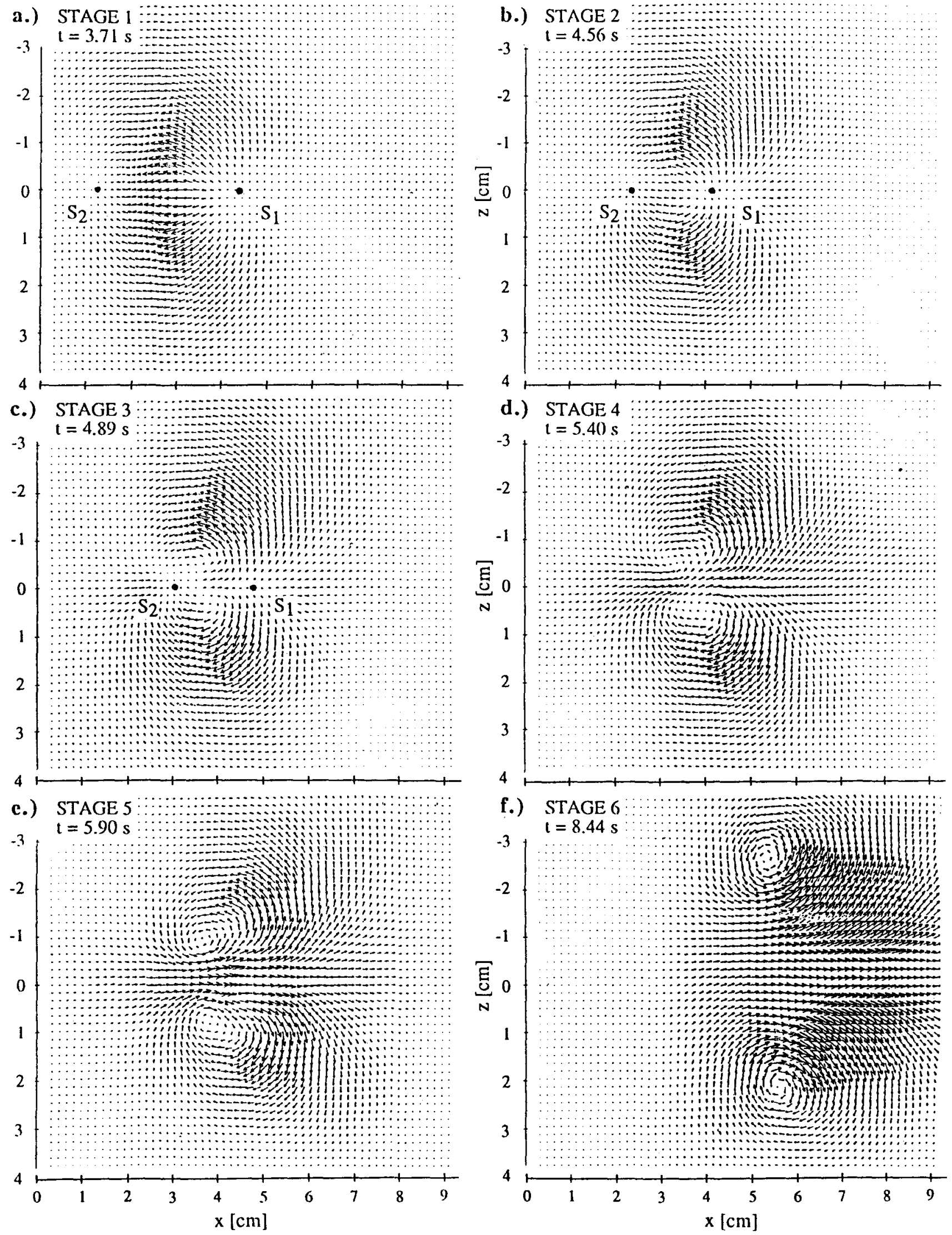

Figure 1a through 1f - Velocity field at the free surface during the reconnection process (Stages 1 to 6 ). 


$$
v\left(\frac{\partial \omega_{z}}{\partial y}\right) \approx-\frac{\partial u_{s}}{\partial t}
$$

It is imperative to appreciate that equation (1) and its approximate form (2) provide a mechanism for the transport of vorticity through the shear-free surface; a mechanism that an inviscid vortex imaging approach could not provide.

By using global velocity measurement techniques such as digital particle image velocimetry (DPIV), we (Gharib et al., 1992) examined various consequences of equation 2 for the case of vortex ring with a Reynolds number of 1000 and a low Froude number of 0.051 . We intentionally chose a low Froude number to keep the deformation of the free surface minimal.

First, we present figure 1 where we mapped the velocity vector field of the approaching vortex ring at a plane $2 \mathrm{~mm}$ below the free surface. Figure la shows the initial motion at the surface due to the induced inviscid velocity field of the approaching vortex (Stage 1). In this early stage, a stagnation point (marked as $S_{1}$ ) appears at $x \approx 41 \mathrm{~mm}$ and $y \approx 0 \mathrm{~mm}$. From this point flow accelerates spatially backward (in the negative $x$ direction) and then through a spatial deceleration converges towards a second stagnation point (marked as $\left.\mathrm{S}_{2}\right)$ at $\mathrm{x} \approx 1.4 \mathrm{~mm}$ and $\mathrm{y} \approx 0 \mathrm{~mm}$. For a normal $\left(\alpha=90^{\circ}\right)$ approach of the vortex ring to a free surface, one would expect a symmetric outward spatial acceleration with a circular stagnation region surrounding $S_{1}$. However, for an oblique approach, only a small arc portion of the primary vortex interacts with the free surface; thus, the observed pattem is a manifestation of the induced velocity of this portion of the vortex ring on the free surface. With time, the size of the stagnation area $\left(S_{1}\right)$ increases and becomes more well defined. However, its location remains unchanged as the second stagnation point moves forward in the positive $x$-direction. Therefore, the flow turns at sharper angles which result in a sink-source (dipole) pattern for the velocity vector field (Fig. 1b). Between Stages 2 and 3, a qualitative change occurs in the flow behavior. At Stage 3 (Fig. 1c), $S_{2}$ moves forward even closer to $S_{1}$ and changes the dipole into a vortex-pair pattern on the surface by reversing the direction of the flow between $S_{1}$ and $S_{2}$ (Stage 4, Fig. 1d). Note that the magnitude of the velocity vectors along the center line (connecting $S_{1}$ and $S_{2}$ ) decreases between Stages 2 and 3 .

With time the vortex-pair pattern is enhanced by a strong forward current at the center which clearly separates the two counter-rotating elongated regions (Fig. 1c). At Stage 5, these elongated regions go through a procession that reshapes them back to circular form. Stage 6 (Fig. 1f) shows completion of the reconnection process and formation of two distinct circular vortices at the surface.

Figure 2 depicts the evolution of the vorticity field in the symmetry plane. These figures show the upper and lower core of the approaching vortex as well as a secondary vortex with its axis parallel to the free surface very near the surface. This secondary vortex with clockwise (negative) vorticity is fairly weak and is located behind and above the upper core of the main vortex. Beyond Stage 2, the vorticity field shows a large vorticity gradient near the free surface marked by the compression of the constant vorticity contours. By Stage 4 , the secondary vortex falls below the noise level of the measurement.

In Figure 3 we depict total circulation of the upper and lower cores of the vortex ring as it approaches

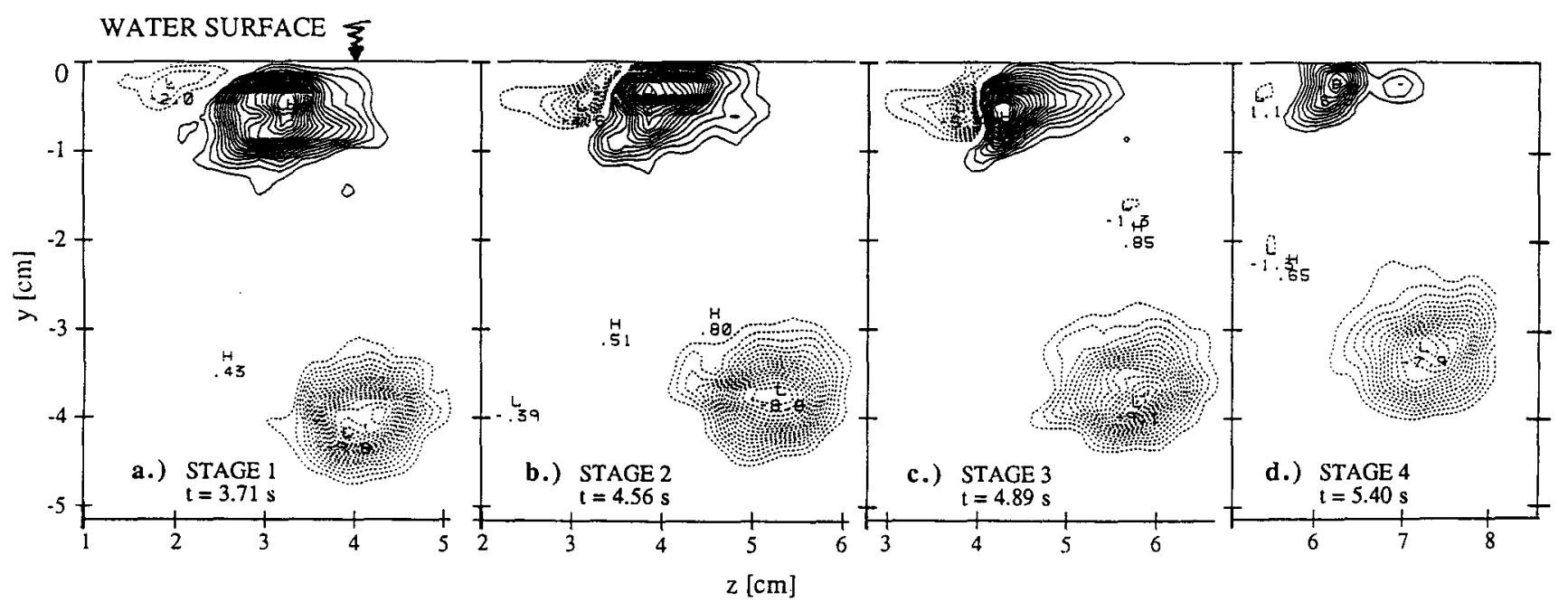

Figure 2: Vorticity field of surface-parallel vortices in the symmetry plane during the disconnection/reconnection process. 


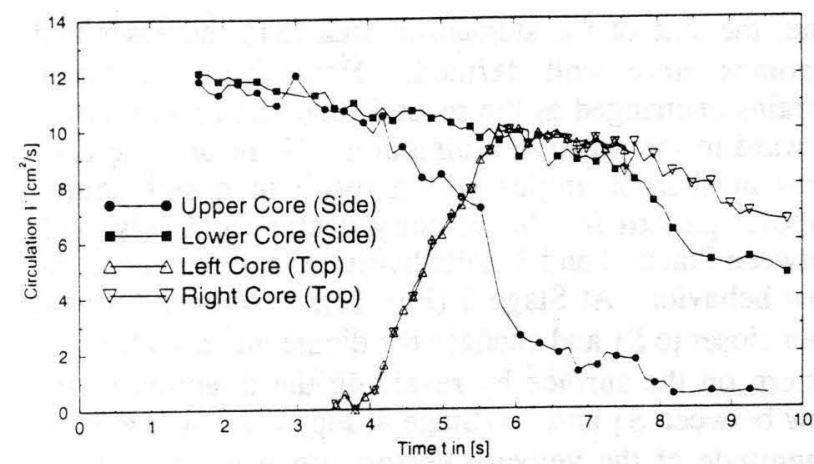

Figure 3: Total circulation $\left(\Gamma\left[\mathrm{cm}^{2} / \mathrm{s}\right]\right)$ of the upper and lower cores of the vortex ring and the reconnecting (left and right of the symmetry plane) vortices to the surface.

the free surface. It is interesting to note the large reduction of circulation for the upper core between stages $2(4 \mathrm{~s})$ and $4(5.5 \mathrm{~s})$ while the lower core goes through a much milder reduction due to the viscous dissipation. Figure 3 also shows the gain of circulation by the newly formed vortices at the surface. It is interesting to note the simultaneous decrease and increase of upper core and surface vortices, respectively.

Prior to Stage (1), the surface velocity along the line that connects $S_{1}$ and $S_{2}$ was accelerated with time to a finite negative value from its initial rest state (i.e. $\left.\partial u_{S} / \partial t<0\right)$. This can be seen in Figure 1a as Stage (1). Therefore, the negative acceleration which results in a positive value for the term $-\partial \mathrm{u}_{\mathrm{S}} / \partial \mathrm{t}$, should initially cause a positive vorticity flux to the region beneath $S_{1}$ and $S_{2}$ which explains the existence of the initial compression of vorticity contour lines near the free surface (Fig. 2). Figure $1 \mathrm{~b}$ shows a reduction in the overall magnitude of the velocity vector with time. This means that $\partial \mathrm{u}_{\mathrm{S}} / \partial \mathrm{t}$ is positive beyond Stage 1 which results in an outward (negative) flux of vorticity through the free surface. The reconnection of surface normal vortices and their induced velocity at the surface is essential in generating a positive $\partial \mathrm{u}_{\mathrm{S}} / \partial \mathrm{t}$ and the reversing of the positive flux process.

\section{INTERACTION OF UNDULATED VORTICES WITH FREE SURFACES}

Another interesting situation arises when a long vortex filament which possesses some undulation approaches a free surface. Unlike the vortex ring case, here the process of interaction is more local and perhaps more typical of vortices in near surface turbulent flows. The situation can be simulated in the laboratory by two counter-rotating undulated flaps. We (Willert, 1992; Willert and Gharib, 1994) studied the interaction of one such modulated pair with free surfaces. The Reynolds number of the vortex pair was about 5,000 and its Froude number about 0.3 . Figures $4 \mathrm{a}$ and $4 \mathrm{~b}$ show one of the modulated vortex filaments before and after its interaction with the surface, where the final stage is typified by a series of half rings connected to the surface. Similar to the vortex ring case, we obtained the circulation of the vortex filament as it evolved with time. As shown in Figure 5, Plane B intersects the core in the middle of the disconnection site.

$$
t=2.00 \mathrm{~s}
$$

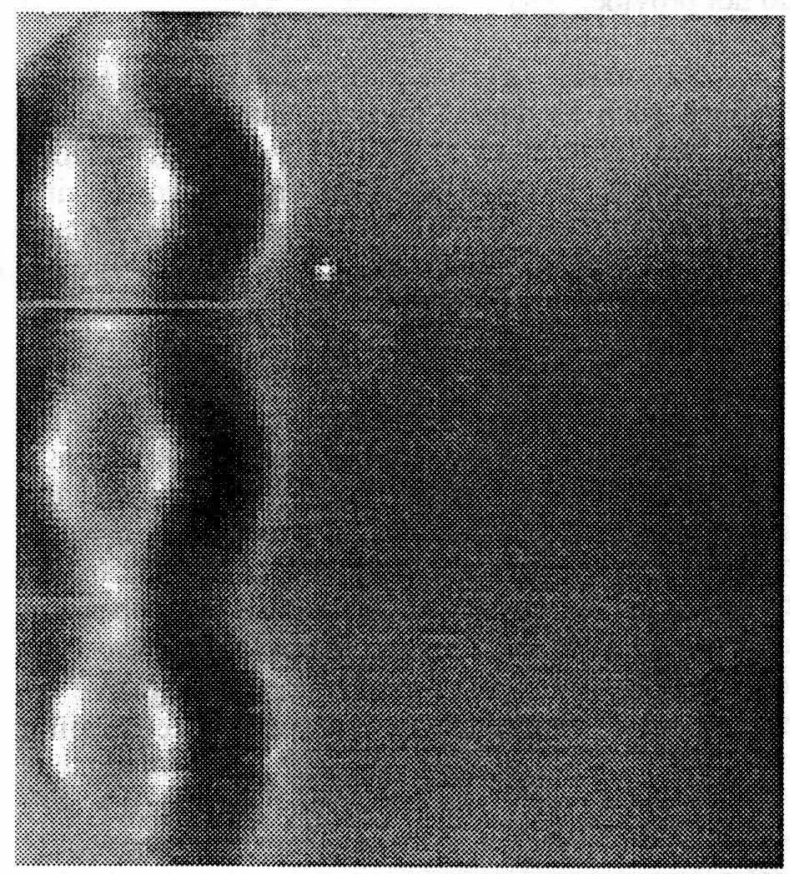

$$
t=3.83 \mathrm{~s}
$$

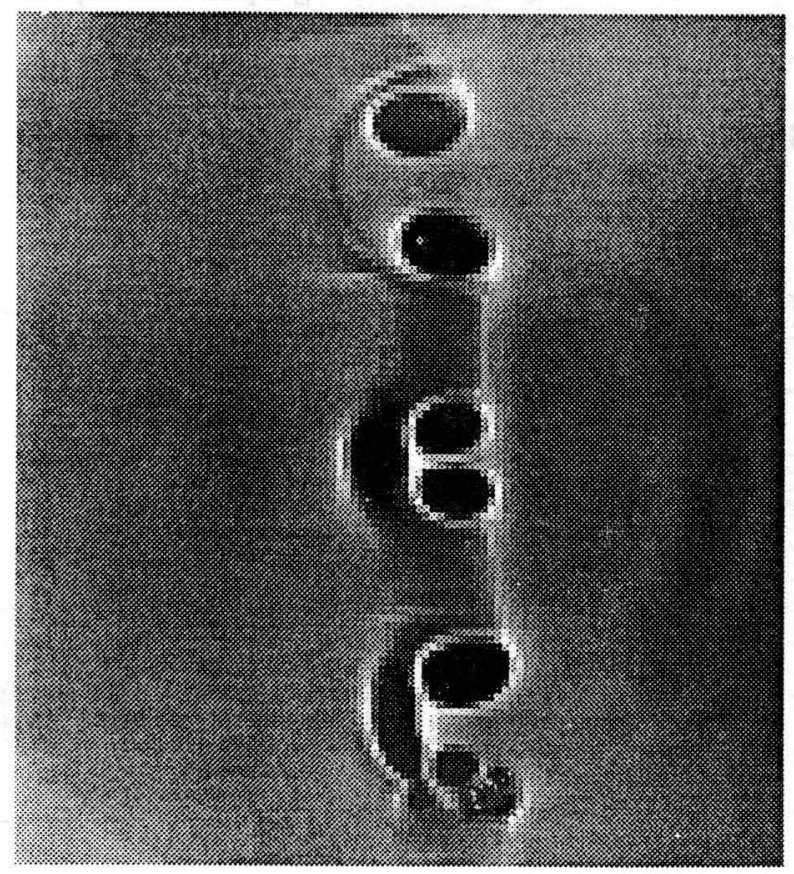

Figure 4: A spatially modulated vortex pair $(\operatorname{Re} \approx 5,000, \mathrm{Fr}$ $\approx 0.3$ ) interacting with a relatively "clean" surface. The core of the vortex moves from the left to the right. 


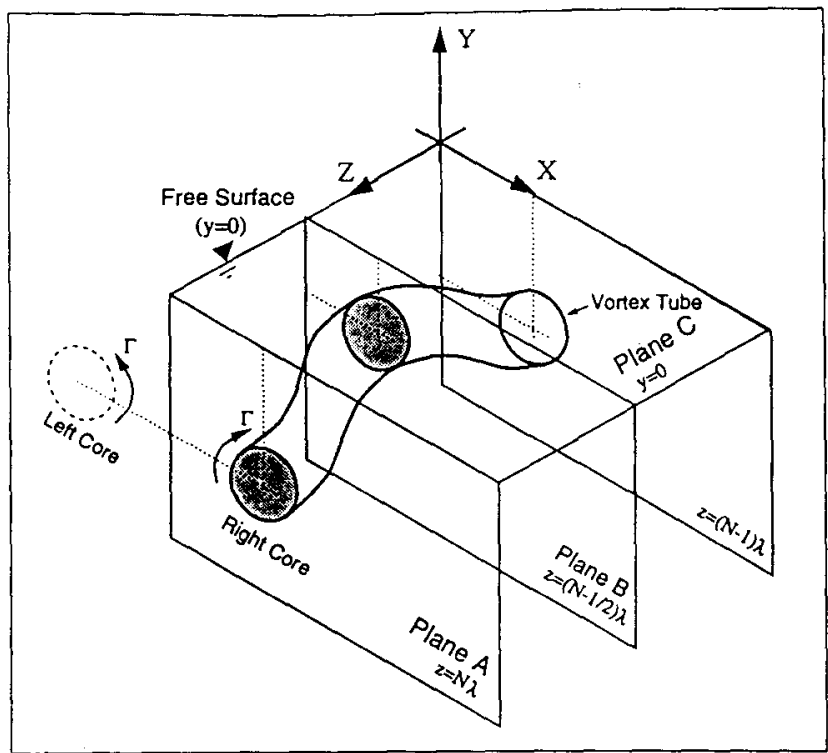

Figure 5: Schematic of the plane of the laser sheets for the DPIV measurements.

The other plane, Plane A, is parallel but removed by half a wavelength. Plane $\mathrm{C}$ consists of the sub-surface plane at approximately $2 \mathrm{~mm}$ below the surface. Figure 6 shows the amount of lost and gained vortices for these three planes. Similar to the vortex ring case, the circulation in Plane B (representing disconnecting surface-parallel vorticity) drops in proportion to the increase of circulation of vortical structures in the sub-surface Plane $C$ (representing reconnecting surface-normal vortices). The circulation of the core region in Plane A remains unaffected throughout the process. A direct check of equation 2 was made by integrating it along a line representing the intersection of Plane A and B with Plane $C$, which expresses the net flux of vorticity per unit depth over the surface. A further integration in time gives a measure of how much circulation $\Delta \Gamma$ is gained or lost. Therefore, $\Delta \Gamma$ can be obtained as follows:

$$
-\Delta \Gamma=\int_{\tau_{0}}^{\tau} \int_{0}^{\infty} \frac{\partial u_{S}}{\partial t} \mathrm{dsd} \tau=\int_{0}^{\infty} \int_{\tau_{0}}^{\tau} \frac{\partial u_{S}}{\partial t} \mathrm{~d} \tau \mathrm{d} d s=\int_{0}^{\infty} \mathrm{usds}
$$

The results of integrating the surface velocity profiles are shown in figure 7. This figure shows that, initially, circulation was created by the surface acceleration up to $t=2$ seconds. Similar to the case of the vortex ring, this is due to the inviscid induced velocity field of an approaching vortex. Along the intersection of $A$ and $C$, where the normal reconnection occurs, the circulation reaches a maximum at time $3 \mathrm{~s}$. This behavior was expected since this portion of the vortex does not interact with the surface.

Interesting is the behavior of surface vorticity along the intersection of $B$ and $C$ where the disconnection occurs. After $\mathrm{t}=2$ seconds, the surface circulation decreases rapidly and would be completely lost.
Temporally, this behavior agrees directly with the circulation measurements obtained from vortices directly. The difference in the maximum values due to undisturbed vortex core $\left(\Delta \Gamma \approx 45 \mathrm{~cm}^{2} / \mathrm{s}\right)$ and the minimum from the latter case (disconnecting core, $\Delta \Gamma \approx 0$ ) is about $80 \%$ of the circulation lost by the near surface core in Plane $A$. This result clearly proves that the surface deceleration is responsible for the vortex disconnection.

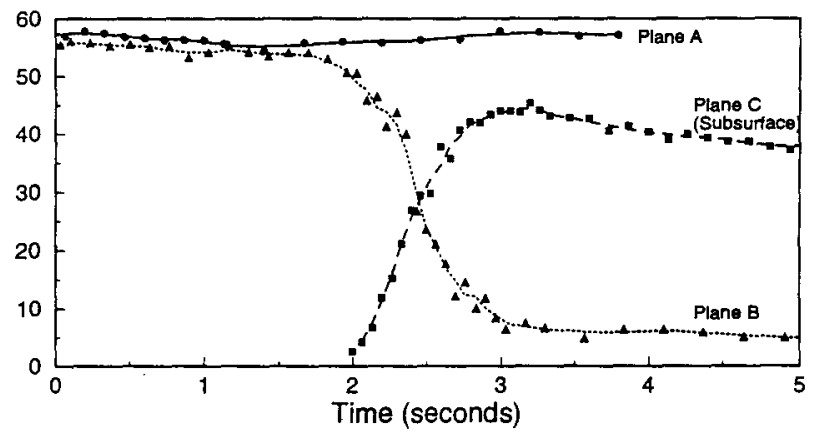

Figure 6: Temporal circulation data obtained from DPIV data in the three imaged planes shown above.

\section{CONCLUDING REMARKS}

It is remarkable that the shear-free condition at the surface results in the reconnection of surface normal vorticity to the surface and a simultaneous disconnection of the surface parallel vorticity due to the outward flux of vorticity through the free surface. The reconnection of surface-normal vorticity to the surface is a requirement imposed by the kinematic condition. However, it appears that such reconnection induces an opposing velocity field to the already existing surface velocity between stagnation points 1 and 2 . Consequently, this opposing induced velocity field decelerates and reverses the flow direction, and, thus, results in a complete disconnection as the surface-normal vortices complete their reconnection to the surface. The reader should be cautioned that the research results reported here are for a relatively clean surface where special care was taken to maintain the surface tension of the water surface constant and near its standard value for pure water.

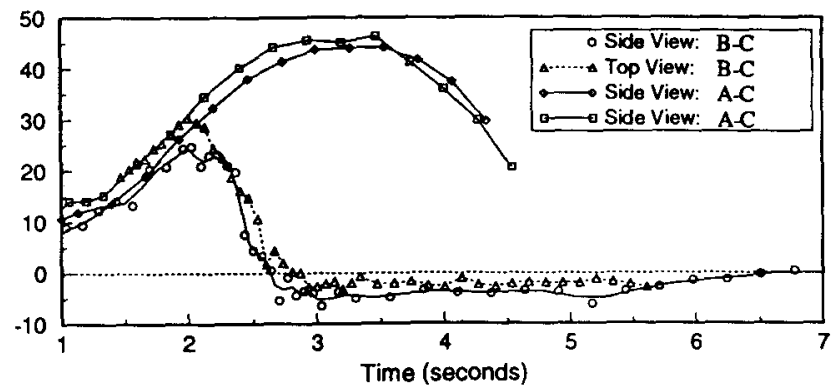

Figure 7: Temporal evolution of surface velocity integrated along a straight line in the surface plane $\left(\int u_{s} d x\left(\mathrm{~cm}^{2} / \mathrm{s}\right)\right)$. 


\section{ACKNOWLEDGMENT}

This paper is based on experiments conducted by Dr. Alexander Weigand and Dr. Christian Willert. The majority of the results for the vortex pairs can be found in Dr. Willert's thesis and in an upcoming paper elsewhere. This work was sponsored by the Office of Naval Research URI grant (N00014-92-J-1610)

\section{REFERENCES}

Roshko, A, Instabilities and turbulence in shear flow, Proceedings of the XVIIIth International Congress of Theoretical and Applied Mechanics, Haifa, Israel, 1992.

Bernal, LP, and Kwon, JT, Vortex ring dynamics at a free surface, Phys. of Fluids A, 1(1989), 449-451.
Rood, EP, Interpreting vortex interaction with a free surface, J. Fluids Engr., (1994a).

Gharib, M, Weigand, A, Willert, CE and Liepmann, D, Experimental studies of a vortex reconnection to a free surface: A physical flow model, Proceedings of the Nineteenth Symposium on Naval Hydrodynamics, Seoul, Korea, 1992.

Willert, CE, and Gharib, M, The interaction of modulated vortex pairs with free surfaces, Proceedings of ASME Symposium of Free-Surface Turbulence, Lake Tahoe, CA, 1994.

Willert, CE, The interaction of modulated vortex pairs with a free surface, Ph.D. Thesis, University of California, San Diego, CA, 1992. 